\title{
Osservazioni astronomiche con veicoli spaziali $(*)$
}

\author{
M. Cimino
}

Ricevuto il 19 Febbraio 1963

RIassuxto. - Si passano in rassegna i più importanti problemi astronomici e astrofisici, e specialmente solari, che attendono un contributo essenziale dalle osservazioni effettuate dai satelliti artificiali. Si auspica che anche in Italia vengano incoragriati i laboratori di ricerca che intendano specializzarsi nella strumentazione spaziale.

Sumary. $-A$ survey is given on the most important astronomical and astrophysical problems, and expecially the solar ones, which need an essential contribution from observations made by artificial satellites. We hope that those research laboratories which intend to develop spatial instrumentation will be supported also in Italy.

\section{INTRODUZIONE.}

1. E questa la seconda volta che ho il piacere di prendere la parola sull'argomento delle osservazioni astronomiche da veicoli spaziali, in un convegno spaziale organizzato dalla Rassegna Elettronica e Nucleare, avendo già parlato su questa materia nel convegno dello scorso anno. Il breve periodo di tempo trascorso è stato però riceo di nuovi studi sull'argomento ed i programmi sono stati sviluppati e precisati, tanto rhe oggi si rende già difficile seguire la vasta bibliografia che appare in materia. Necessariamente questa mia relazione non può essere che una indicazione generale dei principali problemi astronomici che attendono una soluzione dai voli spaziali, per la parte concettuale più che per quella tecnica, della quale ultima si vanno gia interessando alcuni laboratori dei nostri Osservatori Astronomici.

$\left(^{*}\right)$ Nota presentata al $2^{\circ}$ Congresso Internazionale Tecnico Scientifico dello spazio. Roma, 19-23 Giugno 1962. 
Dirò subito che i problemi atmosferici e ionosferici non saramno trattati in questa relazione, giacchè di essi si oceupano in questo stesso convegno eminenti colleghi; così non parlerò del campo magnetico terrestre e delle fasce di radiazione che circondano la terra, né mi oceuperò dei raggi cosnici. Il campo del nostro discorso è quello più rigorosamente astronomico, e riguarderà perciò i seguenti oggetti:

Sole, Luna e pianeti;

Stelle, nebulose galattiche ed estragalattiche;

Spazio interplanetario, interstellare e intergalattico.

2. Talle osservazioni astiononiche con veicoli spaziali la scienza si attende grandi cose; basti pensalve a quanto noi perdiamo nell'osservazione a causa dell'atmosfera terrestre, che può essere classificato come segue:

a) perdita del potere risolutivo dei nostri stmmenti ottici, a causa dell'agitazione atmosferica. Anche nelle condizioni di maggiore calma ed in località particolarmente faroreroli, è paticamente impossibile scendere all di sotto di 0.3 secondi d'arco, e raramente al di sotto del secondo d'arco, specialmente nelle osservazioni solari. Occorre però aggiungere che, per quanto riguarda l'agitazione atmosferica delle immagini, basta raggiungere i $20-30 \mathrm{~km}$ di altezza per avere una calma atmosferica sufficiente per sfruttare in pieno il potere risolutivo degli strumenti oftici. Pertanto in questo genere di osservazioni, strumenti portati da palloni sembrano ancor oggi essere economicamente più convenienti dei satelliti. Già nella mia relazione dello scorso anno ricordai i lanei di Arouin Toollfuss e di Martin Schwarzschild, con i quali è stato portato un prino decisivo contributo al problema della granulazione solare. Come tutti samo, la fotosfera solare, osservata con forti ingrandinenti in momenti di estrema. calma atmosferica, presenta un aspetto gremulare, a "chicehi di riso" delle dimensioni apparenti da 1 a 2 secondi l'areo (circa $1000 \mathrm{~km}$ ), cosa che denota la presenza di uno strato convettivo immediatamente al di sotto della fotosfera. Isa misura delle dimensioni trasversali, della vita media, della velocità e della temperatura dei granuli è essenziale per la comprensione del meccanismo che li determina onde decidere, per esempio, se si tratta di uno stato stazionario del tipo Bénard, orvero se è presente l'intero spettro della turbolenza non stazionaria, o di uno stato intermedio, come le prime osservazioni dello Schwarzsehild sembrerebbero indicare. Molti dati mancano ancora per una piena comprensione teoretical del fenomeno, e molto ai si attende dal nuovo progetto 
dello Schwarzschild di lanciare un telescopio di $90 \mathrm{~cm}$ di apertura e rara finezza di lavorazione, capace di un potere risolutivo di 0.1 secondo d'arco. Oltre al problema della granulazione solare, si attendono da questo esperimento, immagini finissime di nebulose, onde scoprire i primi accenni della nascita di nuove stelle. Accade che in alcune regioni il gas stellare divenga gravitazionalmente instabile; ora circa il 90\% del gas interstellare è formato da idrogeno non ionizzato a bassissima temperatura, dell'ordine di $100^{\circ}$ assoluti, per il quale gas le velocità di turbolenza osservate sono dell'ordine di $10 \mathrm{~km} / \mathrm{sec}$, ossia largamente supersoniche nell'ambiente considerato; da ciò segue la rottura del gas in masse minori, che rappresentano le protostelle. E proprio la scoperta di questi ultimi nuclei di condensazione che interessa l'astronomo. Inutile dire che il telescopio stratosferico potrà dare particolari delle superfici di Venere, Marte e della stessa Luna, di altissimo interesse pei futuri viaggi spaziali.

Naturalmente le osservazioni da bordo di palloni sono effettuate sempre nel campo delle radiazioni visuali, giacchè l'altezza di 20-30 mila metri è insufficiente per lo studio sistematico delle rediazioni ultra-violette. Vorrei sottolineare che osservazioni astronomiche possono essere effettuate utilmente da palloni stratosferici, anche con strumenti assai più modesti del telescopio di Schwarzschild; un riuscito esperimento in tal senso fu da noi effettuato in collaborazione con l'Aeronautica Militare e con l'Istituto Nazionale di Geofisica lo scorso ammo in occasione dell'eclisse totale di sole, ed altri lanei speriamo di poter effet tuare nel prossimo futuro per lo studio della corona solare esterna e della luce zodiacale;

b) la seconda perdita prodotta dall'atmosfera terrestre è quella di larghe bande di radiazioni elettromagnetiche provenienti dai corpi celesti. Per tali radiazioni assorbite, l'altezza di $30 \mathrm{~km}$ è insufficiente; allora è necessario un osservatorio spaziale vero e proprio. Poiché il problema della misura delle radiazioni u. $v$. proveniente dai corpi celesti non può essere effettuato che da bordo di satelliti, appare giustificata la tendenza attuale di destinare quasi esclusivamente a tale scopo i primi osservatori astronomici spaziali, lasciando per ora ai palloni, assai più economici, gli strumenti ad alto potere risolutivo per le fotografie celesti;

c) infine l'atmosfera terrestre, a causa dell'emissione propria di radiazioni, fa si che il fondo del cielo non sia mai, durante la notte, completamente privo di radiazioni spurie. Anche in questo caso, per eliminare completamente dal fondo del cielo le ladiazioni atmosferiche, occorrono grandi altezze e l'uso di osservazioni astronomici su satelliti si rende pertanto indispensabile. 


\section{OSSEIVAZIONI SOLARI.}

3. Prima di passare ad un esame dettagliato delle osservazioni astronomiche da satelliti, ricordiamo il fatto ben noto che le radiazioni elettromagnetiche che raggiungono la Terla sono soltanto una modesta porzione di tutta la gamma esistente. Cominciando con le radiazioni di grande lunghezza d'onda (radionde) è noto come tutte le onde con lunghezza d'onda superiore ai $100 \mathrm{~m}$ circa siano assorbite negli strati più elevati della ionosfera e non penetrano al di sotto dei $700-800 \mathrm{~km}$ circa; anche le onde tra 100 e $10 \mathrm{~m}$ subiscono un fortissimo assorbimento, mentre soltanto le onde dai $10 \mathrm{~m}$ a qualche cm circa possono effettivamente arrivare al suolo. Al di sotto del centimetro interviene l'assorbimento molecolare, particolarmente intenso nella troposfera. Da questo punto in poi, andando verso le frequenze sempre più elevate, dobbiamo arrivare alle ladiazioni luminose, di lunghezza d'onda da $10^{-6} \mathrm{~m}$ in giù, per trovare un'altra "finestra " nella nostra atmosfera, però anche essa assai stretta, giacchè non si estende praticamente al di sotto dei $3200 \AA$, eccezione fatta per una piccola apertura intorno a $2100 \AA$, che noi pensiamo potrebbe forse essere sfruttata con strumenti portati da palloni. Gli agenti maggiolmente responsabili dell'assorbimento sono l'ozono per la banda da 3000 a $2000 \AA$, mentre tra i 2000 e $1000 \AA$ l'assorbimento è causato per la maggior parte dalle molecole dell'ossigeno. Schematizzando grossolamanente, sembra che le radiazioni che controllano gli strati ionosferici siano:

per lo strato $\mathrm{D}: \quad 8 \AA$ del sole attivo; Iyman-alfa del sole quieto;

$$
\begin{aligned}
& " \text { " } " \text { E: } 100 \AA ; \\
& " \text { F: emissione dell'clio nella banda da } 100 \text { a } 500 \AA .
\end{aligned}
$$

E interessante d'altra parte vedere quali sono le zone dell'atmosfera solare dalle quali provengono le radiazioni assorbite dalla nostra atmosfera. E noto che gli astronomi sogliono dividere l'atmosfera solare in tre strati fondamentali: la fotosfera, dalla quale proviene la quasi totalità della radiazione solare, la cromosfera e la corona. Mentre la fotosfera ha una temperatura effettiva di 5750 gradi, - alla quale corrisponde un'emissione che ha il suo massimo nella zona giallo-verde dello spettro, - passando alla cromosfera si verifica un amento progressivo della temperatura che, nella corona, arriva a qualche milione di gradi. Si può dire perciò che la fotosfera solare è la zona piu fredda del Sole 
ed anche quella ove la ionizzazione è minore. Isa cromosfera si può considerare uno strato di passagroio tra la fotosfera e la corona, e non c’è da meravigliarsi perciò se in essa si trovano elementi a bassa temperatura (4000 circa) e altra a temperatura elevata (10.000 gradi), mentre lo spettro da continuo tende a diventare, per la sparizione degli ioni idrogenici negativi, uno spettro a righe di emissione. Intorno a $\lambda 1680$ lo spettro continuo fotosferico diventa trascurabile, mentre importante comincia a diventare quello a righe di emissione della cromosfera. Si può dire perciò che, intorno a tale lunghezza d'onda, si ha una inversione dello spettro solare. E nella cromosfera solare che hanno luogo i brillamenti (i così detti solar-flares) che costituiscono una delle manifestazioni più interessanti dell'attività solare e sono fonte di intense perturbazioni nello spazio interplanetario e nelle atmosfere dei pianeti e della Terra in particolare. Per quanto riguarda infine la corona, mentre nel visuale la sua emissione è 1 milione di volte più piccola di quella fotosferica, nella regione al di sot to dei $100 \AA$, possiamo dire che l'emissione solare ¿̀ dovuta interamente alla corona. Si ha uno spettro continuo doruto ad atomi altamente ionizzati, con massimo attorno ai $30 \AA$ (700.000 gradi in media) ed un ancora più importante spettro a righe, osservato dal gruppo Friedman del Naval Research Laboratory.

Dopo queste brevi premesse, non c'è da meravigliarsi se fino ad oggi assai poco si conosceva direttamente dalla radiazione cromosferica e coronale; quasi tutte le informazioni venivano a noi indirettamente, attraverso lo studio della ionosfera, sensibile alle radiazioni u.v. Uno dei principali compiti dell'osservazione da veicoli spaziali sarà perciò quella della misura sistematica delle radiazioni solari nell'u.v. a nel campo dei raggi $X$, e in particolare uno studio più approfondito dei brillamenti. Riteniamo che tra le altre, le osservazioni più urgenti siano:

I) la misura dei raggi $X$ di $2 \div 6 \AA$, i quali provengono dalle condensazioni coronali che si formano al di sopra dei brillamenti. Tali raggi perturbano fortemente la ionosfera tra i 65 e gli $80 \mathrm{~km}$ di altezza, con assorbimento delle onde radio metriche e riflessione di quelle più lunghe, intensificazione dei disturbi radio atmosferici e crochet geomagnetici;

II) la misura diretta dell'emissione corpuscolare. Il 50\% dei brillamenti sono seguiti da surges entro pochi minuti, con proiezione di materia nello spazio a velocità di molte centinaia di $\mathrm{km}$ al secondo. Questi raggi corpuscolari, attraversando la corona solare, stimolano l'emissione di radionde di frequenza dipendente dallo strato attraversato. Osservando 
il graduale incremento della lunghezza d'onda si può determinare la velocità del raggio corpuscolare; generalmente si osservano due velocità distinte, una intorno ai $1000 \mathrm{~km} / \mathrm{sec}$, l'altra ai $100.000 \mathrm{~km} / \mathrm{sec}$, questa. ultima in concomitanza con brillamenti molto intensi. $A$ questi raggi corpuscolari sono dovute le tempeste geomagnetiche, che si verificano normalmente da 22 a 58 ore dopo il brillamento secondo l'intensità di questi ultimi, — ciò che denota una velocità media dell'ordine dai 9000 a $700 \mathrm{~km} / \mathrm{sec}$-, e le aurore polari con emissione di linee spettrali aurorali in $H_{\alpha}$ e $H_{\beta}$, cosa che conferma velocità dell'ordine dai 400 a $3000 \mathrm{~km}$. Lo studio di questi raggi coronali interessa, oltre che il Sole, anche lo spazio interplanetario; essi sono formati da protoni ed elettroni che si muovono nel plasma interplanetario con velocità supersonica; l'aumento di pressione determinato dall'onda d'urto dovrebbe essere contenuto in uno spazio brevissimo e ciò spiegherebbe la breve durata (2 o 3 minuti) dei così detti "Sudden Commencements" delle tempeste magnetiche; l'onda d'urto, viaggiando ad una velocità di $1500 \mathrm{~km}$, dovrebbe dar luogo a fenomeni luminosi, incluso l'ultravioletto. L'osservazione del fenomeno da bordo di satelliti sarebbe di estremo interesse. La materia proiettata dal Sole trasporta con sé, per noti teoremi di magnetofluidodinamica, anche un campo magnetico; incontrando la Terra i corpuscoli determinano il cosi detto vento solare, misurato direttamente per la prima volta dal satellite Explorer $\mathrm{X}$, portante speciali apparati costruiti clal MIT sotto la direzione del prof. Bruno Rossi. Ricordiamo anche lo correnti corpuscolari emesse da macchie unipolari, con velocità fino a $500 \mathrm{~km} / \mathrm{sec}$;

III) la misura dell'intensità della radiazione Lyman-alfa (prima riga della prima serie spettrale dell'idrogeno). La quasi totalità dell'idrogeno nella fotosfera ì allo stato neutro; pertanto nello spettro solare deve essere particolarmente intensa la prima riga della serie di Lyman per $\lambda 1216 \AA$. Ma essa deve osservarsi in emissione nella corona, anzi è probabile che la radiazione di Lyman-alfa contenga più energia che tutto il resto delle righe di emissione nello spettro tra $976 \AA$ (continuo di Lyman) e i $2000 \AA$. La Lyman-alfa è stata più volte misurata con apparati portati da razzi, ed è stato anche ottenuto qualche spettroeliogramma di tutta la superficie solare in tale riga. Questo genere di osservazione è molto importante per lo studio della corona sul disco; la radiazione fotosferica a 1216 essendo assorbita, rimane in evidenza l'emissione coronale in tale lunghezza d'onda, la quale sembra essere eccitata dai brillamenti. 
Ho accemnato soltanto a qualcuna delle osservazioni solari più interessanti che possono farsi da veicoli spaziali, ma esse non sono che una parte; rolendo riservare un po' del tempo disponibile a parlare delle osservazioni stellari, mi limiterò semplicemente a ricordare l'importanza dello studio delle variazioni spettrali lungo il raggio solare (che richiede però un puntamento di alta precisione), dei profili delle righe spettrali u.v., dei raggi $X$ intorno ai $50 \AA$ (linee di risonamza dei ioni coronali Fe $\mathrm{X}-\mathrm{XV}, \mathrm{Ni} \mathrm{XII}$, ecc.), degli spettroeliogrammi nelle righe di risonanza $\lambda .584$ dell' $H e$ I e $\lambda 304$ dell'He II ed infine delle osservazioni della corona più interna (cosi detta corona $F$ ) e della luce zodiacale, di speciale interesse non solo per la fisica solare, ma anche per lo stadio della materia interplanetaria.

\section{Osservazioni Di STELLE E NEBULOSE.}

4. E passimo ormai alle osservazioni di stelle e nebulose. Diciamo subito che in questo campo è stato fatto quasi nulla. Invece qui si apre una vastissima prospettiva alle ricerche, che investono problemi della più alta importanza, dalla costituzione ed evoluzione delle stelle, ai problemi della forma, dell'origine e dell'evoluzione dell'universo.

È sempre dalle radiazioni di breve lunghezza d'onda (dai 3000 ai $912 \AA$ ) che ci aspettiamo nuove fondanentali informazioni. Per quanto riguarda le stelle, è noto che esse possono avere diverse temperature superficiali, dai 2000 ai 50.000 gradi. Ora noi osserviamo bene lo spettro delle stelle di tipo non molto diverso di quello solare, in quanto il massimo dell'emissione nel loro spettro cade nel visuale. Ma per le stelle molto calde, per es. del tipo $B_{o}$, il massimo di emissione cade intorno ai 1200 $1300 \AA$ e percio la maggior parte dello spettro sfugge ai nostri attuali mezzi di osservazione.

Il campo degli studi stellari da veicoli spaziali è estremamente vasto; mi limitero soltanto ad elencare sommariamente aleuni problemi: osservazione di stelle di tipo solare per confronto delle radiazioni cromosferiche; stelle giganti rosse, per le quali potrebbe attendersi una esagerata cromosfera; flare-stars (stelle a rapidi brillamenti), che incrementano per la durata di pochi minuti la loro luminosità da 10 a 100 rolte; stelle di Wolf-Rayet, (con spettri di emissione), le cui temperature superficiali sono poco note; stelle centrali delle nebulose planetarie; novae e supernovae, dalle quali ultime sembra venir proiettata nello spazio parte della loro materia; ecc. ecc. 
Passando all'osservazione delle nebule, entriamo in un ampo più strettamente legato alla fisica dello spazio, giachè esse sono formate da materia, - gas e polveri - , che occupa in modo più o meno denso gli spazi cosmici. Aleune di queste nebule, - come ad esempio quella di Orione -, rivestono un grande interesse cosmologico, in quanto in esse si trovano quelle associazioni stellari che sembrano strettamente legate alla nascita delle stelle. Abbiamo già detto che queste osservazioni sono fatte meglio con grandi telescopi portati da palloni, ma l'importante studio delle concentrazioni nebulari emittenti luce ultravioletta non potrebbe essere effettuato che da veicoli spaziali. Analogamente è importante lo studio ultravioletto delle atmosfere delle nebulose planetarie che rappresentano, forse, l'altro estremo dell'evoluzione stellare, quello prossimo alla fine. Altre osservazioni su nebule potrebbero interessare la fotometria delle nebulose diffuse e lo studio degli spettri nebulari a righe di emissione.

Materia INTERSTELLARE.

4. Passando alla materia interstellare, è noto che essa sembra costituita di polveri e gas, quest'ultimo principalmente idrogeno, che può trovarsi allo stato atomico neutro, in regioni fredde (dette $H I$ ), 0 ad elevate temperature (regioni $H I I)$, o infine allo stato molecolare. Ise prime regioni possono essere studiate dalla superficie terrestre coi radiotelescopi sull'onda di $21 \mathrm{~cm}$; le seconda pure da terra nella radiazione della riga $H_{a}$ a $\lambda 6563 \AA$; per le nubi di idrogeno molecolare invece, per le quali si hanno diverse indicazioni indirette di esistenza, occorre lavorare nell'ultravioletto, sulla lunghezza d'onda di $\lambda 1284 \AA$, che è una riga di diffusione Raman della Lyman-alfa. E dubbia anche una riga a 2,38 micron. Sfortunatamente, a causa dell'assorbimento dell'idrogeno interstellare, nessuna radiazione emessa da corpi celesti lontani, di lunghezza d'onda pari a quella della $L_{\alpha}$ o più breve del continuo di I yman a $\lambda$ 912, può raggiungere il sistema solare; questa è una grave limitazione, ma d'altra parte possiamo consolarei col dire che i sistemi ottici con finestre al fluoruro di litio, che si usano per radiazioni giù fino a $1100 \AA$, sono in sostanza sufficienti a studiare tutte le radiazioni nltraviolette osservabili nello spazio. Eccezione viene fatta per i raggi $X$, ove si ha una nuova finestra di trasparenza del mezzo interstellare, per l'osservazione dei quali sorgono problemi di natura completamente differente. 
Ma un particolare interesse presenta l'osservazione delle nebulose estragalattiche, specialmente in relazione al problema della materia intergalattica. Quest'ultima è estremamente tenue, ma dato l'enorme volume occupato, la massa totale potrebbe essere cospicua, tanto da alterare sostanzialmente la densità media dell'miverso, stimata dal conteggio delle sole nebulose estragalattiche (una nebulosa per megaparsec cubo). Ora è noto come la densità media della materia nell'miverso sia un dato cosmologico fondamentale, specialmente nei modelli relativistici dell'Universo. Per osservarla un metodo potrebbe essere il seguente: è noto che le galassie distanti presentano un notevole spostamento delle righe spettrali verso il rosso; ora noi abbiamo detto che né la radiazione di Iyyman-alfa, né il continno della serie di Lyman provenienti da stelle può essere osservato, a causa dell'assorbimento dell'idrogeno interstellare della nostra gallassia; ma se la nebulosa estragalattical ha una relocità radiale superiore ai $1000 \mathrm{~km} / \mathrm{sec}$, l'emissione di Lyman risulterà spostata dalla zona di assorbimento e potrà essere osservata dal nostro satellite.

L'osservazione dell'intensità della serie e del continuo di Lyman provenienti da galassie lontane, ci darà una precisa indicazione se lo spazio intergalattico è relativamente libero o meno di idrogeno. In tal modo lo studio delle galassie nella regione u.v. dello spettro potrà fornire cospicue informazioni sulla distribuzione della materia intergalattica.

Sempre con osservazioni dell'emissione Lyman-alfa nelle galassie lontane è possibile studiare anche la distribuzione delle popolazioni stellari.

\section{OSSERVAZIONI A RADIO FREQUENZA.}

6. Abbiamo parlato finora di osservazioni da eflettuare nella zona dello spettro a breve lunghezza d'onda. Ma osservazioni da satelliti possono essere effettuate all'altro estremo, per onde superiori ai 10-15 metri, per la trasmissione delle quali l'atmosfera terrestre costituisce un un ostacolo. Se noi valutiamo la radiazione totale delle stelle riceruta sulla terra in tutto il campo visuale otteniamo un valore dell'ordine di $10^{-8}$ quella del Sole; ma alle basse frequenze madio si ottiene un valore sorprendentemente intenso, circa $10^{4}$ volte quella solare, con ma temperatura equivalente di radiazione sopra i $100.000 \mathrm{~K}$ a circa $10 \mathrm{~m}$ di lunghezza d'onda. Per le alte frequenze la temperatura e l'intensita decrescono, tanto che per frequenze inferiori ai $4 \mathrm{~cm}$ risulta assai piccola; 
non sembra perciò che l'osservazione da veicoli spaziali di radioonde al di sotto del centimetro sia particolarmente interessante. Al contrario sarebbero di estrema importanza osservazioni sul campo superiore ai 15 metri. Ammettendo una densità elettronica di 600 elettroni al $\mathrm{cm}^{3}$ nello spazio interplanetario, i satelliti dovrebbero poter esplorare la gamma delle frequenze fino ai 220 kilocicli: per frequenze ancora più basse, la materia interplanetaria ne impedirebbe l'arrivo.

Lascerò questo pur vasto campo delle osservazioni nelle radio frequenze limitandomi a ricordare l'importanza dell'osservazione extraatmosferica dei radio "burst " solari e di quelli provenienti dal pianeta Giove, i quali ultimi suggeriscono l'esistenza di una ionosfera, con un campo magnetico dell'ordine di due gauss.

\section{Cosmotogri.}

7. Un dato di capitale importanza per la cosmologia consiste nella misura della radiazione integrale proveniente da tutto il cielo. Sulla superficie terrestre tale misura è falsata sia dall'assorbimento, sia dalla luminosità propria dell'atmosfera.

Naturalmente occorrerà stabilire un opportuno programma per separare la percentuale di luce proveniente da oggetti galattici da quella degli oggetti extragalattici. Se, per esempio, l'Universo fosse euclideo, infinito e senza espansione, con distribuzione media delle galassie uniforme, la brillanza del cielo dovrebbe risultare infinita e noi tutti ci troveremmo in questo momento al centro di un forno. Il fatto che ciò non accade è assai signifieativo. In ogni caso, qualumque sia il modello di universo, la distribuzione delle galassie potrà supporsi più o meno uniforme e la brillanza del cielo potrà, di conseguenza, essere dedotta e confrontata con l'osservazione.

Oltre a ciò, le più recenti osservazioni della brillanza superficiale delle galassie mostrano che tali agglomerati di stelle, anziché presentare un orlo definito, vanno sfumando nello spazio; ciò fa supporre l'esistenza nello spazio intergalattico non solo di gas e polveri, ma forse anche di stelle. Sarebbe assai interessante perciò poter misurare la brillanza assoluta del cielo nelle regioni intergalattiche, cosa che dalla superficie terrestre è impossibile, essendone impediti, come si è detto, dalle radiazioni e dall'assorbimento proprio dell'atmosfera.

Sempre restando in campo cosmologico, aceennerò appena alla possibile verifica della variazione della frequenza di un oscillatore immerso in un 
campo gravitazionale. Questo effetto, previsto dalla Relatività Generale, dà notoriamente uno spostamento verso il rosso della lunghezza d'ondal delle raliazioni solari osservate dalla Terra; se, al contrario, l'oseillatore si trovasse su un satellite, dovrebbe aversi, prescindendo da ogni eventuale effetto Doppler, uno spostamento verso il violetto, dato che l'oscillatore verrebbe a trovarsi un campo gravitazionale meno intenso di quello in cui si trova l'osservatore. Risultando il rapporto $\frac{\Delta v}{v}$ dell'ordine di $7 \times 10^{-10}$, misure dell'effetto relativistico non sarebbero possibili nel campo delle radiazioni visuali, ma lo sarebbero invece in quello delle radionde, dato che la stabilità dei campioni di frequenza atomici e molecolari è appunto dell'ordine di $10^{-9}-10^{-10}$.

Vorrei infine accennare ad una ricerca di enorme valore speculativo: quella di eventuali spore vaganti nello spazio, per confermare o meno la ben nota ipotesi della panspermia, della propagazione eioè della vita dell'universo. Tenendo presente che esistono microrganismi capaci di resistere per tempi lunghissimi anche in alto vuoto e a temperature bassissime, e che la loro sezione d'urto alle radiazioni di altissima energia ò relativamente piccola, il loro principale nemico resterebbe sempre lat radiazione u.v. solare e quella corpuscolare protonica. Ma un piecolo strato di materia potrebbe essere sufficiente a schermarli. Un esperimento attivo di panspermia è stato pure proposto, di lanciare cioè microorganismi terrestri nello spazio e constatarne la sopravvivenza.

\section{SATELLITI ASTRONOMICI.}

8. Naturalmente molte altre osservazioni potranno essere fatte dai satelliti artificiali; non ho parlato, per esempio, dell'osservazione della nostra stessa Terral effettuata dal di fuori. Gli strati superiori dell'annosfera terrestre intereagiscono non solo con la radiazione corpuscolare ionizzata, ma anche con le radiazioni elettromagnetiche. Così, ad es. si ha una diffusione della radiazione Lyman-alfa per risonanza, la quale dovrebbe perció apparire fortemente riflessa dall'atmosfera. Osservazioni analoghe potrebbero esser eseguite sulle atmosfere degli altri pianeti da sonde spaziali.

Ma il tempo stringe e dobbiamo spendere aneora qualche parola sull'organizzazione degli osservatori astronomici spaziali.

Per quanto riguarda le osservazioni solari, i dati fino ad oggi pubblicati provengono da razzi e da satelliti portanti piceoli strumenti per 
l'ultravioletto e i laggi $\mathrm{X}$. Ho pure aceemmato all'impresa dell'Explorer $\mathrm{X}$ per lo studio diretto del plasma interplanetario e del cosi detto vento solare. Dal 7 Maggio ca. è in ordita il satellite solare OSO (Orbiting Solar Observatory), del (quale tutti arrete potuto leggere una descrizione sulle numerose riviste specializzate o di divulgazione. L'orbita del satellite è inchinata di 33 gradi sull'equatore, con un apogeo di $395 \mathrm{~km}$, un perigeo di 547 ed un periodo di rivoluzione di 96 minuti cirea.

Il secondo osservatorio astronomico spaziale sarà probabilmente quello del progetto Celescope, e costituirà il primo satellite portante telescopi veri e propri, destinati alle osservazioni stellari. Questo satellite dovià, tra l'altro, trasmetterei ma mappa generale del cielo in una larga banda dell'ultravioletto, contenente circa 100.000 stelle; ogni puntata coprirà 2,2 gradi quadrati, e ne occorreranno, per' coprire l'intero cielo, 8520 opportunamente distribuiti in cirea 400 giorni complessivi di osservazione. Tutto il sistema del Celescope è costruito attorno and una camera televisiva provvista di più tubi sensibili a diverse bande spettrali, alla lunghezza d'onda effettiva di $2200 \AA ; 1500 \AA ; 1350 \AA$, con ma soglia di sensibilità pari a una stella di $10^{\mathrm{a}}$ grandezza. Il tempo massino di integrazione di tali detector è dell'ordine di 30 secondi, ma esistono già tubi vidicon con tempi di integrazione fino a 1000 secondi. Il potere risolutivo non sara molto loutano dai $30^{\prime \prime}$ di areo); pochissime (appena l'uno per cento) saramo perciò le stelle doppie che apparimanno sdoppiate.

Il Celescope porterà quattro camere del tipo di Schwarzschild, di $30 \mathrm{~cm}$ di apertura ciaseuna; una di esse dovrebbe funzionare da spettrografo senza fenditura, avendo al posto del primo specehio un reticolo conaro con 200 linee al $\mathrm{mm}$, orientato in modo che sul cumpo del detector Cada la banda spettrale ra 1100 e $2200 \AA$.

Il sutellite trasmetterà via radio le osservazioni, secondo una suecessione temporale prestabilita.

Tra i veicoli spaziali in progetto dobbiamo fare particolare menzione delle cosi dette sonde spaziali (probe), specialmente di quelle destinate ad attraversare la corona solare a distanza relativamente ravvicinata al sole. Oceorre a tal proposito tener presente che a 4 raggi solari dal sole si hamno temperature radianti di 3000 gradi e ciò può dare un indicazione della complessità dei problemi tecnici che attendono i rostruttori delle sonrle solari.

9. Naturalmente, sono moltissimi i problemi tecenici che bisogna affrontare nella realizzazione di un osservatorio astronomico spaziale; le riviste specializzate trattano diffusamente di questo argomento sul quale 
non è possibile entrare in una relazione a carattere generale. Vorrei soltanto chindere questa mia chiacchiepata con alcune considerazioni che potrebbero servire di orientamento per l'organizzazione delle nostre ricerche nel campo della progettazione e della costruzione di strumenti astronomicei per veicoli spaziali.

Vediamo innanzi tutto a quale altezza devono ruotare gli osservatori astronomici spaziali. Per lo studio dello spettro u.x. del sole potrebbe bastare un'altezza dai 350 ai $500 \mathrm{~km}$, alla quale però l'assorbimento dell'ozono, ossigeno e idrogeno possono ancora risultare importanti. Per' le osservazioni della Lyman-alfa una attenuazione potrebbe teoricamente sussistere anche oltre i $2000 \mathrm{~km}$, a causa del sospettato involucero di idrogeno che, secondo alcuni autori, cinconderebbe la terra. Una decisione è impossibile prima che un sufficiente materiale di osservazione non sia stato raceolto; si può però ritenere che la quasi totalità delle osservazioni nella radiazione Lyman-alfa possono essere effettuate, almeno provvisoriamente, anche da altezze superiori ai 200 km soltanto.

Ma, certamente, un osservatorio posto a maggiore altezza dei normali satelliti di due orè è preferibile; per es. un satellite di 24 ore $(35,560 \mathrm{~km})$ sarebbe assai conveniente, perchè sarebbero assai facilitate la trasmissione dei dati, mentre la terra apparirebbe sotto un angolo di meno di $20^{\circ}$ e sarebbe possibile stabilire un sistema di riferimento stellare orientato su tre stelle immutabili.

Riguardo ai telescopi, accenneremo alle ricerche in corso per costruire specehi leggeri di quarzo con nervature o anche di leghe o metalli leggeri (alluminio, titanio, invar a berillio); un telescopio di apertum dell'ordine di $1.20 \mathrm{~m}$ darebbe già un potere risolutivo superiore a quello ottenuto oggi sulla terra, tenendo conto della scintillazione atmosferica; il suo peso, con specchio de] tipo leggero sopradetto e con tutti gli accessori necessari al funzionamento, potrebbe essere contenuto nei limiti della capacità di trasporto di un vettore del tipo Saturno. Una difficoltà sorge nella lavorazione dello specechio, il quale dovendo lavorare anche per le radiazioni n.v., dovrebbe avere una superficie lavorata con un'acecuratezza notevolmente superiore a quella ordinaria. Durante il puntamento è richiesta una stabilita dell"ordine del secondo di areo, che però deve scendere a 0.1 durante le esposizioni.

Riteniamo che la progettazione di strumenti per satelliti richieda la creazione di veri a propori laboratori specializzati; in questo senso noi stessi intendiamo muoverei. Questi laboratori dovrebbero essere, a loro volta, in intimo contatto con l'industria; negli Stati Uniti le più importanti fabbriche di aerei, - ora interessate alla costruzione di missili 
(Lockheed, Douglas, ...), - hamno creato laboratori scientifici di ricerche spaziali. Molte idee originali si vanno sviluppando nei nostri laboratori; per dotare i futuri nostri satelliti di strumenti pin efficienti ed originali, occorre dare ai nostri ricercatori la possibilità materiale e creare l'ambiente favorevole per sviluppare i loro ritrovati. Ora bisogna pensare che, se è pur vero che i problemi di lancio hamno una grande importanza tecnica e industriale, è altrettanto vero che la soluzione dei problemi più strettamente scientifici, fisici ed astronomici, non può essere postecipata al raggiungimento del primo scopo. Sarebbe una grave mortificazione se, per sviluppare le nostre idee sulla strumentazione astronomica per satelliti, doressimo attendere il primo lancio. I due problemi hamno la stessa importanza e vamno affrontati contemporaneamente. 